\title{
Negative mass as a source of gravity
}

\author{
Open Physics Collaboration*† \\ June 13, 2020
}

\begin{abstract}
We propose that negative mass can be the source of gravity in the quantum vacuum.
\end{abstract}

keywords: negative mass, curvature, vacuum, quantum gravity

The most updated version of this paper is available at https://osf.io/k4gtz/download

\section{Source of negative curvature}

1. Positive mass/energy curves spacetime [1].

2. Negative mass/energy curves spacetime.

3. Curvature is due to sources of gravity (matter/energy).

4. Suppose the vacuum state of our universe is described by Anti-de Sitter space (AdS) [2].

5. AdS has negative curvature.

*All authors with their affiliations appear at the end of this paper.

†Corresponding author: mplobo@uft.edu.br | Join the Open Physics Collaboration 
6. So, according to (4) and (5), our spacetime has negative curvature.

7. If the curvature is negative, then a source of negative mass/energy is fulfilling our universe.

8. The source (7) is provided by quantum mechanics, that is, the quantum properties of spacetime (vacuum).

\section{Mass entanglement}

9. Conjecture: positive mass is entangled with negative mass.

10. Let

(a) $|q\rangle=$ quantum vacuum state

(b) $|+\rangle /|-\rangle=$ state of positive/negative energy

(c) $m_{+} / m_{-}=$amplitude of the positive/negative mass

11. $|q\rangle=m_{+}|+\rangle+m_{-}|-\rangle$

12. Breaking the entanglement (11) leads to free negative energy [3].

\section{Final Remarks}

13. Classical vacuum states might be the result of quantum phenomena, such as: spacetime pouring [3], Hawking radiation applied to ordinary matter [4], dark matter [5], and other effects [6].

\section{Open Invitation}

Review, add content, and co-author this paper [7,8]. Join the Open Physics Collaboration. Send your contribution to mplobo@uft.edu.br. 


\section{Ethical conduct of research}

This original work was pre-registered under the OSF Preprints [9], please cite it accordingly [10]. This will ensure that researches are conducted with integrity and intellectual honesty at all times and by all means.

\section{References}

[1] Misner, Charles W., Kip S. Thorne, and John Archibald Wheeler. Gravitation. Princeton University Press, 2017.

[2] Witten, Edward. "Anti de Sitter space and holography." arXiv preprint hep-th/9802150 (1998). https://arxiv.org/pdf/hepth/9802150.pdf

[3] Lobo, Matheus P. "Spacetime Pouring." OSF Preprints, 21 May 2019. https://doi.org/10.31219/osf.io/zwfb5

[4] Lobo, Matheus P. "Ordinary Matter and Dark Energy." OSF Preprints, 25 May 2019. https://doi.org/10.31219/osf.io/ersvu

[5] Lobo, Matheus P. "Dark Matter and Bubbles of Void." OSF Preprints, 11 July 2019. https://doi.org/10.31219/osf.io/w7m3q

[6] Lobo, Matheus P. "Gravity Extracts Virtual Particles from the Quantum Vacuum (davies-fulling-unruh Effect)." OSF Preprints, 19 May 2019. https://doi.org/10.31219/osf.io/tq7m2

[7] Lobo, Matheus P. "Microarticles." OSF Preprints, 28 Oct. 2019. https://doi.org/10.31219/osf.io/ejrct

[8] Lobo, Matheus P. "Simple Guidelines for Authors: Open Journal of Mathematics and Physics." OSF Preprints, 15 Nov. 2019. https://doi.org/10.31219/osf.io/fk836 
[9] COS. Open Science Framework. https://osf.io

[10] Lobo, Matheus P. "Negative Mass as a Source of Gravity." OSF Preprints, 26 July 2019. https://doi.org/10.31219/osf.io/k4gtz

\section{The Open Physics Collaboration}

Matheus Pereira Lobo (lead author, mplobo@uft.edu.br),1

David Adjei ${ }^{2}$

${ }^{1}$ Federal University of Tocantins (Brazil); ${ }^{2}$ Kwame Nkrumah University of Science and Technology (Ghana) 